Case Reports

\title{
Primary Eosinophilic Lung Diseases and the Therapeutic Role of Corticosteroids: A Case Report and Literature Review
}

\author{
${ }^{1}$ Huzairi Sani and ${ }^{2}$ Nada Syazana Zulkufli \\ ${ }^{1}$ Faculty of Medicine, Universiti Teknologi MARA, Selangor 68100, Malaysia \\ ${ }^{2}$ Department of Pathology, Penang General Hospital, Pulau Pinang, Malaysia
}

\author{
Article history \\ Received: 05-12-2019 \\ Revised: 13-01-2020 \\ Accepted: 08-02-2020 \\ Corresponding Author: \\ Huzairi Bin Sani \\ Faculty of Medicine, Universiti \\ Teknologi MARA, Selangor \\ 68100, Malaysia \\ Email: huzairis@gmail.com
}

\begin{abstract}
A 17-year-old girl was seen and treated for eosinophilic pneumonia with underlying Hypereosinophilic Syndrome (HES), a rare disorder comprising of the triad: A persistently high eosinophil count, eosinophil-mediated organ damage and with no evidence of other causes of secondary eosinophilia. The diagnosis was subsequently changed to acute exacerbation of chronic eosinophilic pneumonia following reviews of journal updates. Pulmonary eosinophilia is a life-threatening condition which is treatable if detected early. Unfortunately, being a rare and poorly understood disease, its diagnosis is often missed and management delayed. The aim of this report is to consolidate the understanding of CEP diagnostics and the therapeutic role of steroids based on available literature.
\end{abstract}

Keywords: Acute Eosinophilic Pneumonia, Chronic Eosinophilic Pneumonia, Eosinophilia, Eosinophilic Lung Disease, Hypereosinophilic Syndrome, Primary Eosinophilic Lung Disease, Pulmonary Eosinophilia, Steroid

\section{Introduction}

\section{Case Report}

A previously well 17-year-old Malay girl was seen in the emergency room for chronic productive cough and progressive shortness of breath for 5 months with no constitutional symptoms. There was no history of exposure to Tuberculosis (TB), travel to endemic areas or consumption of medications within the last year. There was no personal or family history of atopy, asthma, pulmonary TB or lung diseases. Social history was unremarkable.

Clinical examination revealed a febrile female who was breathing using her accessory muscles at a rate of 60 breaths/minute. She was also tachycardic at 110 beats per minute with a blood pressure of $120 / 70 \mathrm{mmHg}$ and oxygen saturation of $71 \%$ on room air. On auscultation of the lungs, there was generalized rhonchi with reduced air entry on the right lung.

An arterial blood gas examination showed type 1 respiratory failure and her chest X-ray demonstrated areas of consolidation with air bronchograms over the entire right lung, a cavitation over the left middle zone and patchy opacities over the left lower zone. She had a white cell count of $18.60 \times 10^{9} / \mathrm{mL}$ with an increased Absolute Eosinophilic Count (AEC) of $4.0-5.0 \times 10^{9} / \mathrm{mL}$ which persisted over the following weeks of admission.
A blood smear did not show leukemic features. Cultures from blood, pulmonary secretions and urine came back negative; sputum studies for mycobacteria were also negative; and immunological studies including AntiNeutrophil Cytoplasmic Antibody (ANCA) were negative. A Computerised Tomogram (CT)-thorax reported areas of consolidation, ground glass opacities, peribronchial thickening and hilar lymphadenopathy features of which were suggestive of Chronic Eosinophilic Pneumonia (CEP). No tissue samples were available for histological or cytological review.

She was treated as CEP with Hydrocortisone following which, significant clinical improvement was seen by day 3 of admission. She was subsequently discharged on day 11 with oral Prednisolone after completing 10 days of parenteral steroids. Prior to discharge, a prophylactic dose of oral Albendazole was given and a repeat radio-imaging of her chest showed considerable clearing.

\section{Review of Literature}

Chronic eosinophilic pneumonia is a rare disorder categorized under the primary eosinophilic lung diseases (ELD) along with acute eosinophilic pneumonia (AEP), hypereosinophilic syndrome (HES) and Churg Straus syndrome (CSS) (Fernandez Perez and Frankel, 2013). Since its first identification in 1960, 
little is still known of its pathogenesis given its rarity. However, there have been advancements in diagnostics and treatment in the last 10 years.

\section{Epidemiology}

There is currently no statistical report of the disease's incidence in Malaysia. However, an American survey covering 10 pediatric centres in 2001 reported 4 cases of CEP in the adolescent population, with a male-to-female ratio of 1:1 (Wubbel et al., 2003). Another three surveys in various parts of Europe covering years 1900 to 2004 reported a heterogeneous incidence rate of CEP, ranging from 0.23 to 7 per 100,000 persons (Coultas et al., 1994; Sveinsson et al., 2007; Thomeer et al., 2001). In the adult population, women, non-smokers, asthmatics and those aged 30-40 years are more commonly affected (Fernandez Perez and Frankel, 2013).

\section{Etiology}

Eosinophils have been strongly implicated in the pathogenesis of the disease where a high number of eosinophils and its related cytokines like IL-3, IL-5, IL6, IL-10 and Granulocyte-Macrophage ColonyStimulating Factor (GM-CSF) were confined to the sections of infiltrated lung whilst sparing the serum and healthy lung tissues (Alam and Burki, 2007; Alberts, 2004). These cytokines, especially IL-5, activate the eosinophil, prevent apoptosis and stimulate degranulation (Fernandez Perez and Frankel, 2013; Alam and Burki, 2007; Uckan et al., 2001).

T-helper 2 cells (Th2) also play a pivotal role where they infiltrate lung interstitium in chemotactic response to RANTES and Thymus-and-Activation-RegulatedChemokine (TARC) and produce IL-5, hence enhancing eosinophilic recruitment to the lungs (Alam and Burki, 2007). These eosinophils, in return, secrete IL-2 which further activates Th2 and causes a continuous cycle of eosinophilic degranulation (Brito-Babapulle, 2003).

Additionally, as elevated $\mathrm{IgE}$ and positive rheumatoid factor are not uncommonly found in CEP, an immunological basis of its pathology is probable (Alberts, 2004).

\section{Diagnostic Workup}

ELD was first defined by Chusid et al. (1975) by 3 criteria: (1) AEC of $>1.5$ for at least 6 months (2) evidence of eosinophil-mediated organ involvement and (3) no underlying disease that causes tissue eosinophilia. The first criterion has been loosened by Roufosse and Weller (2010) to two readings of AEC of $>1.5$ taken a month apart as patients may primarily present during an exacerbation requiring timely treatment to avoid endorgan damage (Helbig, 2013; Dulohery et al., 2011).

Underlying diseases known to cause eosinophilia should be ruled out such as infections, parasitic infestations, allergic diseases, drugs, lung diseases, connective tissue diseases, skin diseases and primary malignancies (Fernandez Perez and Frankel, 2013; Brito-Babapulle, 2003; Lim et al., 2014). It is also imperative to rule out myeloid malignancies by peripheral blood film analysis, vitamin B12 assay, FIP1L1 PDGFRa fusion gene analysis, bone marrow biopsy and cyogenetics. Erythrocyte Sedimentation Rate (ESR) and C-reactive protein have been found to be of little help owing to their lack of specificity and sensitivity (Fernandez Perez and Frankel, 2013).

To confirm lung involvement, pulmonary tissue from either Bronchoalveolar Lavage (BAL) or lung biopsy is sampled along with radiological commodities. In healthy patients, it is normal to find $<2 \%$ eosinophils in the lungs (Alam and Burki, 2007). The presence of $>40 \%$ eosinophils along with lymphocytes, plasma cells, polymorphonuclear neutrophils in the BAL are suggestive of pulmonary eosinophilia although $>25 \%$ of eosinophils is enough to make a suspicion (Fernandez Perez and Frankel, 2013; Alam and Burki, 2007; Brito-Babapulle, 2003). Elevated IL-5, TARC and RANTES in the BAL are also suggestive of CEP as these are not found in other interstitial lung diseases (Alam and Burki, 2007). However, it is important to note that BAL is not helpful in distinguishing between the different ELDs and other interstitial lung diseases. Definitive confirmation is by histological evidence of eosinophils within the alveoli. Despite that, lung biopsy is seldom required unless clinical, radiological and laboratory cues are non-confirmatory (Fernandez Perez and Frankel, 2013). Thoracic CT scans yield variable findings in ELD, given the heterogeneous etiology of the disease, although a ground glass appearance is characteristic of a eosinophilic infiltrative process (Fernandez Perez and Frankel, 2013; Dulohery et al., 2011).

As CEP and AEP are strictly confined to the lungs, any systemic eosinophil-mediated inflammatory damage favours the diagnoses of HES or CSS (Fernandez Perez and Frankel, 2013; Brito-Babapulle, 2003; Lim et al., 2014). Lung involvements have been reported in 24-40\% of patients with HES (Dulohery et al., 2011; Lim et al., 2014) but the extent of eosinophilia in the lung tissues of HES is not as marked as in CEP and AEP (Tefferi et al., 2006). On the CT thorax, patchy ground glass opacities and consolidation lean towards HES as opposed to the more peripherally-distributed opacities and the classical "photographic negative of CCF" prevalent in CEP (Alam and Burki, 2007; Kim et al., 1997).

The onset of CEP is more sinister in comparison to the acute onset of AEP, with the former occurring over a period of weeks to months. Symptoms for both entities are similar albeit of a milder degree in CEP and that preexisting bronchial asthma is more prevalent in CEP 
rather than AEP (Alam and Burki, 2007). Patients with AEP usually present in acute respiratory distress, which is rarely seen in CEP unless an exacerbating event is present (Fernandez Perez and Frankel, 2013; Alam and Burki, 2007). Some striking features distinguishing the two lie in the diagnostic workup, where in CEP, a persistent peripheral eosinophilia of $>10 \%$ is characteristic as opposed to the infrequently mild or even absent peripheral eosinophilia in AEP (Alam and Burki, 2007; Dulohery et al., 2011; Tefferi et al., 2006). Chest $\mathrm{X}$-ray of CEP usually shows bilateral, migratory opacities located peripherally, giving a photographic negative of pulmonary edema whereas in AEP, the opacities are more diffuse bilaterally and associated with pleural effusions (Alam and Burki, 2007; Tefferi et al., 2006). Similarly on CT thorax, peripherally-located patchy ground glass consolidations indicate CEP, whilst diffusely spread ground glass appearance with interlobular septal thickening and pleural effusions allude to AEP (Dulohery et al., 2011; Kim et al., 1997). BAL may not help in discriminating CEP from AEP as both show marked eosinophilia. However, a lung biopsy demonstrating organizing diffuse alveolar damage and edema, on top of eosinophilic infiltration, are suggestive of AEP (Alam and Burki, 2007). The lung function test in CEP progresses from normal to obstructive to restrictive patterns according to the disease's chronicity whereas the pattern in AEP is consistently restrictive (Dulohery et al., 2011). While both pneumonias respond dramatically to steroids, AEP only requires less than 2 months' course of steroid therapy while CEP requires treatment for over 6 months (Fernandez Perez and Frankel, 2013; Plutinsky et al., 2007). Spontaneous recovery from AEP have been reported, suggesting that response to steroid therapy may not be diagnostic for AEP (Jhun et al., 2015; Philit et al., 2002). Lastly, relapses do not occur in AEP and this isn't the case with CEP (Alam and Burki, 2007).

\section{Role of Steroids in Management}

Glucocorticoids disrupt cytokine signalling between white cells, suppress colony formation and regulate cell surface receptors that receive regulatory molecules. These cumulatively reduce eosinophilic count, shorten cellular lifespan and prevent degranulation (Uckan et al., 2001; Brito-Babapulle, 2003). It has been reported that eosinophilic asthmatics show better control with inhaled corticosteroids than non-eosinophilic asthmatics (Martin et al., 2011).

Another study compares the response of two siblings with hypereosinophilia to different doses of prednisolone where the sibling on a single High Dose Methylprednisolone (HDMP) showed significant AEC reduction in comparison to the sibling on conventional prednisolone. This suggests that HDMP alters the phenotype of eosinophils and reduces their activity (Uckan et al., 2001).

It has been reported that CEP patients who are already on inhaled steroids for asthma had a lower relapse rate of $23 \%$ compared to $56 \%$ amongst CEP patients not on inhaled steroids. Another small-scale study reported out of 5 patients with CEP on both oral and inhaled steroids, 3 were able to have their maintenance dose of prednisolone tapered (Alam and Burki, 2007). However, inhaled steroids are not effective when prescribed as monotherapy and should be given in conjunction with oral steroids for symptomatic resolution (Minakuchi et al., 2003). There was only one unusual case report of a woman already on long-term prednisolone for rheumatoid arthritis who presented with AEP but dramatically improved with a single dose of HDMP (Shin et al., 2013).

Response to treatment is reflected by symptomatic recovery, radiological resolution, eosinophilic count reduction and lung function test improvement. Three separate studies following the progress of CEP patients over 4, 6.2 and 10 years respectively suggested increased relapse rates with time (Marchand et al., 1998; Durieu et al., 1997; Naughton et al., 1993). It was also concluded that a minimum of $15 \mathrm{mg} / \mathrm{kg} / \mathrm{day}$ of steroid is needed for maintenance (Alam and Burki, 2007). Nonetheless, despite it being the mainstay treatment, alternative therapies have also been explored to achieve the lowest remission dose.

Other non-steroidal therapies include cytotoxic agents like cyclosporine which inhibits IL-2 transcription factor and $\alpha$-interferon which disrupts eosinophils at the stem cell level (Fernandez Perez and Frankel, 2013; Brito-Babapulle, 2003). Hydroxyurea is reserved for steroid-resistant cases at a dose of 1-2 g/day whilst vincristine, chlorambucil and etoposide are reserved for intractable end-organ damage as these drugs risk inducing myelodysplastic syndromes and secondary leukemia (Brito-Babapulle, 2003). Considering the crucial role of IL-5 in the pathophysiology of eosinophilic diseases, anti-IL-5 monoclonal antibodies are currently being studied (Antoniu, 2010). Also recently, 2 cases of CEP were successfully treated with 2-weekly injections of anti-IgE therapy for 5 months. Both patients subsequently had their oral corticosteroids stopped and maintained on inhaled steroids without relapses after at least 15 months (Shin et al., 2012).

\section{Study Limitations}

While the patient outcome was good, earlier detection and commencement of steroids could have shortened her hospital stay. Diagnostically, no histopathological study was available although its absence did not affect treatment and clinical outcome. 


\section{Conclusion}

It is important to rule out all causes of eosinophilia and distinguish CEP from other ELDs as treatment vary between diagnoses. The mainstay treatment for CEP remains to be steroids (HDMP in the acute setting and low-dose oral prednisolone during maintenance) although other alternatives are currently being studied.

\section{Acknowledgment}

We thank everyone involved in this case report for their contributions. We extend our utmost thanks to the Internal Medicine team members, participants and staff of Selayang Hospital, Selangor, Malaysia.

\section{Author's Contributions}

Huzairi Sani: Involved manuscript writing, case management and literature review.

Nada Syazana: Involved in writing, proofreading and critical revision of the manuscript.

\section{Ethics}

Informed consent has been obtained from the case study and anonimity is preserved in this case report.

\section{References}

Alam, M. and N.K. Burki, 2007. Chronic eosinophilic pneumonia: A review. Southern Med. J., 100: 49-53. DOI: $10.1097 / 01 . s m j .0000242863 .17778 .1 \mathrm{~d}$

Alberts, W.M., 2004. Eosinophilic interstitial lung disease. Curr. Opin. Pulmonary Med., 10: 419-424. DOI: 10.1097/01.mcp.0000130330.29422.8d

Antoniu, S.A., 2010. Novel therapies for hypereosinophilic syndromes. J. Med., 68: 304-310.

Brito-Babapulle, F., 2003. The eosinophilias, including the idiopathic hypereosinophilic syndrome. Brit. J. Haematol., 121: 203-223.

DOI: 10.1046/j.1365-2141.2003.04195.x

Roufosse, F. and P.F. Weller, 2010. Practical approach to the patient with hypereosinophilia. J. Allergy Clin. Immunol., 126: 39-44.

DOI: 10.1016/j.jaci.2010.04.011.

Chusid, M.J., D.C. Dale, B.C. West and S.M. Wolff, 1975. The hypereosinophilic syndrome: Analysis of fourteen cases with review of the literature. Medicine (Baltimore), 54: 1-27.

DOI: 10.1097/00005792-197501000-00001

Coultas, D.B., R.E. Zumwalt, W.C. Black and R.E. Sobonya, 1994. The epidemiology of interstitial lung diaseses. Am. J. Respir. Crit. Care Med., 150: 967-972. DOI: 10.1164/ajrccm.150.4.7921471
Dulohery, M.M., R.R. Patel, F. Schneider and J.H. Ryu, 2011. Lung involvement in hypereosinophilic syndromes. Respiratory Med., 105: 114-121. DOI: 10.1016/j.rmed.2010.09.011

Durieu, J., B. Wallaert and A.B. Tonnel, 1997. Long term follow-up of pulmonary function in chronic eosinophilic pneumonia. Eur. Respir. J., 10: 286-291. DOI: $10.1183 / 09031936.97 .10020286$

Fernandez Perez, E.R. and S.K. Frankel, 2013. Eosinophilic lung diseases. Clin. Pulmonary Med., 20: 280-291. DOI: $10.1097 / C P M .0000000000000009$

Helbig, G., 2013. Advances in the diagnosis and treatment of eosinophilia. Curr. Opin. Hematol., 21: 3-7. DOI: 10.1097/MOH.0000000000000011

Jhun, B.W., S.J. Kim, R.C. Son, H. Yoo and B. Jeong et al., 2015. Clinical outcomes in patients with acute eosinophilic pneumonia not treated with corticosteroids. Lung, 193: 361-367. DOI: $10.1007 / \mathrm{s} 00408-015-9722-\mathrm{X}$

Kim, Y., K.S. Lee, D Choi, S.L. Primack and J. Im, 1997. The spectrum of eosinophilic lung disease: Radiologic findings. J. Comput. Assisted Tomography, 21: 920-930. DOI: 10.1097/00004728-199711000-00015

Lim, K., J. Ko, S.S. Lee, B. Shin and D. Choi et al., 2014. A case of idiopathic hypereosinophilic syndrome presenting with acute respiratory distress syndrome. Allery Asthma Immunol. Res., 6: 98-101. DOI: 10.4168/aair.2014.6.1.98

Marchand, E., M. Reynaud-Gaubert, D. Lauque, J. Durieu and A.B. Tonnel et al., 1998. Idiopathic chronic eosinophilic pneumonia: A clinical and follow-up study of 62 cases. Medicine, 77: 299-312. DOI: 10.1097/00005792-199809000-00001

Martin, N., C.E. Brightling and I.D. Pavord, 2011. Eosinophils best marker of steroid response. Thorax, 66: 730-730. DOI: 10.1136/thx.2010.144592

Minakuchi, M., A. Niimi, H. Matsumoto, R. Amitani and M. Mishima, 2003. Chronic eosinophilic pneumonia: Treatment with inhaled corticosteroids. Respiration, 70: 362-366. DOI: 10.1159/000072898

Naughton, M., J. Fahy and M. Fitzgerald, 1993. Chronic eosinophilic pneumonia: a long-term follow-up of 12 patients. Chest, 103: 162-165.

DOI: $10.1378 /$ chest.103.1.162

Philit, F., B. Etienne-Mastroianni, A. Parrot, C. Guerin and D. Robert et al., 2002. Idiopathic acute eosinophilic pneumonia: A study of 22 patients. Am. J. Respiratory Critical Care Med., 166: 1235-1239. DOI: $10.1164 / \mathrm{rccm} .2112056$

Plutinsky, J., D. Magula, S Petricek, D. Laukova and S. Majernik et al., 2007. Acute and chronic eosinophilic pneumonia: Our own experience with diagnosis and treatment. World Allergy Organiz. J. DOI: 10.1097/01.WOX.0000302160.00553.11 
Shin, H.Y., J.W. Choe, M. Kwon, J.Y. Jang and J.W. Jung et al., 2013. Acute eosinophilic pneumonia leading to acute respiratory failure in current systemic corticosteroid user. Allergy Asthma Immunol. Res., 6: 242-244. DOI: 10.4168/aair.2013.5.4.242

Shin, Y.S., H.J. Jin, H. Yoo, Y.H. Nam and Y. Ye et al., 2012. Successful treatment of chronic eosinophilic pneumonia with anti-IgE therapy. J. Korean Med. Sci., 27: 1261-1264.

DOI: $10.3346 / \mathrm{jkms} .2012 .27 .10 .1261$

Sveinsson, O.A., H.J. Isaksson and G. Gudmundsson, 2007. CEP in Iceland: Clinical features, epidemiology and review. Laeknabladid, 93: 111-116.

PMID: 17277407

Tefferi, A., M.M. Patnaik and A. Pardanani, 2006. Eosinophilia: Secondary, clonal and idiopathic. Brit. J. Haematol., 133: 468-492.

DOI: $10.1111 / \mathrm{j} .1365-2141.2006 .06038 . \mathrm{x}$
Thomeer, M.J., U. Costabe, G. Rizzato, V. Poletti and M. Demedts, 2001. Comparison of registries of ILD in 3 European countries. Eur. Respir. J. Suppl., 32: 114s-118s. PMID: 11816817

Uckan, D., G. HIcsonmez, B. Tunc, M. Cetin and I. Tezcan et al., 2001. The analysis of eosinophil and lymphocyte phenotype following single dose of high-dose methylprednisolone in two siblings with marked hypereosinophilia. Clin. Lab. Haem., 23: 33-37. DOI: 10.1046/j.1365-2257.2001.00345.x

Wubbel, C., D. Fulmer and J. Sherman, 2003. Chronic eosinophilic pneumonia: A case report and national survey. Chest, 123: 1763-1767.

DOI: $10.1378 /$ chest.123.5.1763 